\title{
Using high-fidelity simulation to design a course for overseas doctors in managing common medical, ethical and legal challenges within the NHS
}

\author{
Authors: Dhanya Pillai and Patrick Tran
}

\begin{abstract}
Aims
To use high-fidelity simulation to prepare overseas doctors with essential technical and non-technical skills when dealing with common medical, ethical and legal dilemmas.
\end{abstract}

\section{Methods}

We surveyed doctors who were new to the NHS to identify challenging workplace situations that are often not covered in trust induction or undergraduate training. Overseas doctors at various grades may not always have senior supervision on these initial encounters, putting patient safety and ultimately fitness to practice at risk. Based on these discussions, we identified four major themes: protecting patient safety within the pressures of bed shortages; decision on escalation care plans in a critically unwell patient without mental capacity; management of needlestick injury and patient confidentiality in high-risk patients; and acting on a serious patient incident and communicating it to relatives as a duty of candour.

These concepts were then incorporated into four medical scenarios, which involved a high-fidelity manikin and simulated actors. The scenarios were initially evaluated by the simulation faculty to ensure they met intended objectives and were practical to run. Once the scenarios were finalised, we piloted a 1-day course to six international candidates with additional workshops and lectures to address key common issues highlighted by the simulation debriefs. Evaluation was collated using a questionnaire with a mixture of open and closed questions graded on a 6-point Likert scale.

\section{Results}

Feedback received was extremely positive. All (100\%) of the candidates confirmed that the course was relevant to their clinical role, praising the scenarios to be clinically relevant and realistic. A common theme among all candidates was realising the importance of non-technical skills, particularly situational awareness, prioritisation and task management, and how ethico- legal issues are addressed in medical emergencies. For example, $83 \%$ felt competent to comply with duty of candour in the face of a serious patient incident.

All $(100 \%)$ candidates agreed that the course was a necessary adjunct to their trust induction, and $100 \%$ either moderately or strongly agreed that they felt 'more confident in commencing a job in the NHS'.

\section{Conclusion}

The course targets discrepancies in the induction of overseas doctors, reflected by cross-cultural differences. It provides an opportunity to manage difficult ethico-legal scenarios and allows revision of skills in a safe learning environment. Hence, the course name, REFRESH (revise and enhance from roleplay ethical scenarios in hospital). We have since integrated this into trust induction and introduced the course to trainees returning to practice.

\section{Conflict of interest statement}

None declared. 